\title{
Handbuch diagnostische Radiologie
}

Herausgeber:

JÜrgen FreysChMidT, BREMEN 
A. STÄBLER (Hrsg.)

Handbuch

diagnostische Radiologie

\section{Muskuloskelettales System 3}

Mit Beiträgen von:

G. Adam, A. Baur-Melnyk, H. M. Bonél, R. Erlemann, J. Freyschmidt, J. Hodler, S. Höpfner, H. Imhof, F. Kainberger, M. Krötz, G. M. Lingg, T. Link, C. Nolte-Ernsting, K.-J. Pfeifer, T.Rand, A.Stäbler, M. Steinborn, U.Szeimies, K. Trieb

Mit 492 Abbildungen in 941 Einzeldarstellungen 
PD Dr. med. AxEL STÄBLER

Radiologie in München Harlaching

Grünwalder Straße 72

81547 München

Titelbild:

Osteopetrose, „,curved“ MPR einer MDCT-Untersuchung (mit freundlicher Genehmigung von Dr. Ulrike Szeimies, München)
ISBN-10 3-540-24229-5

Springer Berlin Heidelberg New York

ISBN-13 978-3-540-24229-1

Springer Berlin Heidelberg New York

Bibliografische Information Der Deutschen Bibliothek

Die Deutsche Bibliothek verzeichnet diese Publikation in der Deutschen Nationalbibliografie; detaillierte bibliografische Daten sind im Internet über <http://dnb.ddb.de $>$ abrufbar

Dieses Werk ist urheberrechtlich geschützt. Die dadurch begründeten Rechte, insbesondere die der Übersetzung, des Nachdrucks, des Vortrags, der Entnahme von Abbildungen und Tabellen, der Funksendung, der Mikroverfilmung oder der Vervielfältigung auf anderen Wegen und der Speicherung in Datenverarbeitungsanlagen, bleiben, auch bei nur auszugsweiser Verwertung, vorbehalten. Eine Vervielfältigung dieses Werkes oder von Teilen dieses Werkes ist auch im Einzelfall nur in den Grenzen der gesetzlichen Bestimmungen des Urheberrechtsgesetzes der Bundesrepublik Deutschland vom 9. September 1965 in der jeweils geltenden Fassung zulässig. Sie ist grundsätzlich vergütungspflichtig. Zuwiderhandlungen unterliegen den Strafbestimmungen des Urheberrechtsgesetzes.

Springer ist ein Unternehmen von

Springer Science+Business Media

springer.de

(C) Springer-Verlag Berlin Heidelberg 2005

Die Wiedergabe von Gebrauchsnamen, Handelsnamen, Warenbezeichnungen usw. in diesem Werk berechtigt auch ohne besondere Kennzeichnung nicht zu der Annahme, dass solche Namen im Sinne der Warenzeichen- und MarkenschutzGesetzgebung als frei zu betrachten wären und daher von jedermann benutzt werden dürften.

Produkthaftung: Für Angaben über Dosierungsanweisungen und Applikationsformen kann vom Verlag keine Gewähr übernommen werden. Derartige Angaben müssen vom jeweiligen Anwender im Einzelfall anhand anderer Literaturstellen auf ihre Richtigkeit überprüft werden.

Planung: Dr. U. Heilmann, Heidelberg

Redaktion: D. Mennecke-Bühler, Heidelberg

Herstellung: PRO EDIT GmbH, Heidelberg

Umschlaggestaltung: Frido Steinen-Broo, EStudio,

Calamar, Spanien

Satz und Reproduktion: AM-productions GmbH, Wiesloch

SPIN 21/3151 Di- $54 \begin{array}{lllll}4 & 3 & 2 & 1 & 0\end{array}$

Gedruckt auf säurefreiem Papier 


\section{Vorwort}

Eine fortlaufende Optimierung der bildlichen Darstellung krankhafter Organveränderungen erfordert ein sich ständig verbreiterndes medizinisches Wissen.

Ein Handbuch ist der Definition nach ein zusammenfassendes, in der Regel mehrbändiges Werk über eine Wissenschaft oder ein spezielles wissenschaftliches Gebiet. Kann ein solches Werk noch Bestand haben in einer Zeit, in der sich wissenschaftliche Erkenntnisse mit nahezu unvorstellbarer Geschwindigkeit entwickeln und wandeln?

Die Herausgeber und Autoren dieses Handbuchs bejahen diese Frage; sie halten es geradezu für notwendig, eine fundierte Standortbestimmung über die diagnostische Radiologie in einem Rahmen abzugeben, der für die praktischen Belange dieses - neben der klinischen Pathologie - wichtigsten diagnostischen Schlüsselfachs prinzipiell einen Wertbestand von etwa 8-10 Jahren besitzen soll. Dabei wurde bedacht, dass sich in diesem Zeitraum zwar untersuchungstechnische Modalitäten, besonders bei der MRT und CT, durchaus ändern werden, dass aber das Prinzip der Darstellungsmöglichkeiten von krankhaften Veränderungen bestimmter Organe oder Organsysteme weitgehend unverändert bleibt; denn die den Krankheiten zugrunde liegenden pathologischanatomischen Veränderungen selbst ändern sich ja kaum!

Die rasche Entwicklung und den Wandel von ätiologischen, pathogenetischen und therapeutischen Erkenntnissen kann und muss man in wissenschaftlichen Zeitschriften und ggf. aktuellen Monographien verfolgen; doch wird man das Neue nur dann verstehen und nutzen können, wenn man durch einen soliden Wissensfundus darauf vorbereitet ist. Dazu soll dieses Handbuch mit seinem besonderen Konzept der Wissensvermittlung beitragen. Es orien- tiert sich an Organen oder Organsystemen mit ihren Erkrankungen, die jeweils bestimmte radiologische Untersuchungsstrategien erfordern (z. B. mit Hilfe der Projektionsradiographie, CT, MRT, Ultraschall, ggf. Szintigraphie).

In den jeweiligen Hauptkapiteln findet sich zunächst eine Darstellung der Normalanatomie und ihrer wesentlichen Varianten - bezogen auf die einzelnen Darstellungsmodalitäten; dann folgt ein Kapitel über die systematische Bildanalyse. Die Kapitel über die einzelnen Krankheitsentitäten (Fehlbildungen, traumatische und entzündliche Veränderungen, Tumoren und sonstige Störungen) sind einheitlich nach folgenden Themen aufgebaut:

- pathologisch-anatomische Grundlagen (zum Verständnis der radiologischen Befunde),

- klinische Symptomatik,

- charakteristische radiologische Symptome und ihre Differentialdiagnose.

- Jedes Kapitel schließt mit Empfehlungen zur Untersuchungsstrategie und zusammenfassenden Merksätzen.

Der rote Faden, der sich durch das gesamte Werk zieht, ist die synoptische Betrachtungsweise von klinischen und mit Hilfe der Radiologie erkennbaren pathologisch-anatomischen und funktionellen Veränderungen. Eine dem Patienten nützliche Diagnostik kann im Übrigen nur aus der Fusion von technischer Entwicklung und einem angepassten medizinischen Wissen um das Wesen und die Vielfalt von Krankheiten gelingen.

Im Frühjahr 2001

Für die Herausgeber und Autoren J. FreYsChMidT, Bremen 


\section{Vorwort}

Die Darstellungsmöglichkeiten des muskuloskelettalen Apparates sind durch die MRT enorm erweitert worden. Damit hat sich auch das Spektrum von Krankheitsentitäten, die der Radiologe kennen sollte, erheblich verbreitert. Deshalb gilt: „Seeing better must be paired with knowing more."

Mit dieser Entwicklung, die zwangsläufig in eine zunehmende Subspezialisierung in der muskuloskelettalen Diagnostik führt, hat sich gleichzeitig eine Veränderung der radiologischen Untersuchungsstrategien bei den einzelnen Krankheitsentitäten vollzogen, der es Rechnung zu tragen gilt. Der Aufbau der Kapitel folgt der bewährten Grundstruktur der anderen Bände dieses Handbuches diagnostische Radiologie (pathologisch-anatomische Grundlagen, klinische Symptomatik, radiologische Symptome und ihre Differentialdiagnose).

Band 3 des Muskuloskelettalen Systems hat als Hauptthemen die systemischen Knochenerkrankungen, die entzündlichen und degenerativen Gelenkerkrankungen und Erkrankungen der Weichteile. Unter Verwendung eines hervorragenden Bildmaterials werden die angeborenen und erworbenen sklerosierenden Knochenerkrankungen, die metabolischen und hämatologischen Systemerkran- kungen, trophische Störungen inclusive Nekrose und schließlich in einer Synopsis Erkrankungen des Periosts dargestellt. Allein das Zusammentragen des kostbaren Bildmaterials dieser z.T. sehr seltenen Erkrankungen hat viele Jahre in Anspruch genommen.

Das umfangreiche Kapitel über die rheumatischen Erkrankungen bewahrt die Tradition der subtilen projektionsradiographischen Diagnostik, verfeinert und vertieft durch aktuelle Ergebnisse einer magnetresonanztomographischen Weichteil- und Knochendiagnostik. Die Kapitel über die (Kristall-)Arthropathien leiten zu den degenerativen Gelenk- und Wirbelsäulenerkrankungen über, die einen wesentlichen und zunehmenden Stellenwert in der radiologischen Diagnostik einnehmen. Beschlossen wird der letzte Band Muskuloskelettales System durch ausführliche Kapitel zu Erkrankungen der Weichteile einschließlich ihrer Tumoren.

Wir, die Autoren, wünschen uns, dass die vorliegenden muskuloskelettalen Bände über die Lösung konkreter diagnostischer Probleme hinaus Lesefreude bereiten und Motivation zur weiteren Vertiefung in diese Thematik der diagnostischen Radiologie hervorrufen.

Im März 2005
Für die Autoren

PD Dr. MEd. A. STÄBLER, München 


\section{Inhalt}

9 Systemische Skeletterkrankungen

9.1 Angeborene sklerosierende Knochenerkrankungen 1

T. LINK, J. FREYSCHMIDT

9.1.1 Osteopoikilie 1

9.1.2 Osteopathia striata 2

9.1.3 Melorheostose 2

J. FREYSCHMIDT

9.1.4 Gemischtförmige sklerosierende

Knochendysplasie 14

9.1.5 Pachydermoperiostose 14

Literatur 16

9.2 Erworbene sklerosierende Knochenerkrankungen 16

T. LiNK

9.2.1 Sekundäre hypertrophische

Osteoarthropathie 16

9.2.2 Infantile kortikale Hyperostose 18

9.2.3 Hyperostosis frontalis interna 19

9.2.4 Chronisch venöse Insuffizienz 19

9.2.5 Diffuse idiopathische

Skeletthyperostose 21

Literatur 25

9.3 Stoffwechselerkrankungen des Knochens 25

T. LINK

9.3.1 Osteoporose 25

9.3.1.1 Formen der Osteoporose 41

9.3.2 Osteomalazie 45

9.3.3 Hyperparathyreoidismus 47

9.3.4 Renale Osteopathie 50

9.3.5 Hypoparathyreoidismus 52

9.3.6 Pseudohypoparathyreoidismus 52

9.3.7 Osteopathie bei Hypo-/Hypervitaminose 53

9.3.8 Hormonelle Osteopathien 54

9.3.9 Toxische Osteopathien 57 Literatur 59
9.4 Reaktive und stressbedingte

Knochenerkrankungen, belastungsbedingte Erkrankungen der Sehnen und der Sehnenansätze 62 A. STÄBLER, M. STEINBORN

9.4.1 Morbus Sudeck 62

M. STEINBORN

9.4.2 Transiente Osteoporose 65

M. STEINBORN

9.4.3 Erkrankungen der Sehnenansätze

(Enthesiopathien) 68

A. StÄBLER, M. STEINBORN

9.4.3.1 Schultergürtel 69

9.4.3.2 Ellenbogengelenk 74

9.4.3.3 Handgelenk 77

9.4.3.4 Becken 78

9.4.3.5 Kniegelenk 82

9.4.3.6 Sprunggelenk und Fuß 85 Literatur 91

9.5 Knochennekrosen 95

G. AdAm, C. Nolte-Ernsting

9.5.1 Pathogenese 95

9.5.2 Osteonekrosen beim Erwachsenen 96

9.5.2.1 Hüftkopfnekrose 96

9.5.2.2 Lunatumnekrose (Morbus Kienböck) 100

9.5.2.3 Femurkondylennekrose (Morbus Ahlbäck) 101

9.5.2.4 Andere Osteonekrosen 103

9.5.3 Osteonekrosen beim Kind 104

9.5.3.1 Femurkopfnekrose (Morbus Perthes) 104

9.5.3.2 Osteonekrose des Capitulum humeri (Morbus Panner) 107

9.5.3.3 Osteonekrose der Tibiaapophyse (Morbus Osgood-Schlatter) 108

9.5.3.4 Andere Osteonekrosen 109

9.5.4 Osteochondrosis dissecans 109

9.5.5 Empfehlungen zur Untersuchungsstrategie bei Osteonekrosen 113

Literatur 113 
9.6 Knochenveränderungen durch hämatologische und retikuloendotheliale Systemerkrankungen 117

A. BAUR-MELnYK

9.6.1 Anämien 117

9.6.1.1 Hämoglobinopathien 117

9.6.1.2 Andere Anämien 122

9.6.2 Plasmozytom/Plasmazelldyskrasien 123

9.6.3 Leukämien 134

9.6.4 Osteomyelofibrose/Osteomyelo-

sklerose 138

9.6.5 Knochenveränderungen bei Erkrankungen des retikuloendothelialen Systems 142

9.6.5.1 Lipidspeicherkrankheiten 142

9.6.5.2 Glykogenspeicherkrankheiten 146

9.6.5.3 Histiozytosen 146

9.6.5.4 Mastozytose 150

Literatur 152

9.7 Systemische Osteoarthropathien 154

T. RAND

9.7.1 Phakomatosen 154

9.7.1.1 Neurofibromatose 155

9.7.1.2 Tuberöse Sklerose 161

9.7.1.3 Zerebelloretinale Hämangio-

blastomatose 164

9.7.1.4 Enzephalotrigeminales Syndrom 164

9.7.2 Sarkoidose 165

9.7.3 Amyloidose 168

9.7.4 Angiodysplasien des Knochens 170

Literatur 173

9.8 Erkrankungen des Periosts 175

R. ERLEMANN

9.8.1 Periostreaktionen 175

9.8.2 Juxtakortikale Läsionen 177

9.8.3 Periostitis 177

9.8.4 Posttraumatische Periostitis 183

9.8.5 Tumoren 187

9.8.6 Tumorähnliche Läsionen 189

Literatur 190

10 Erkrankungen der Gelenke

10.1 Rheumatoide Arthritis und juvenile Arthritiden 191

G.M. LINGG, H.M. BoNÉL

10.1.1 Rheumatoide Arthritis 191

10.1.2 Juvenile Arthritiden 218

10.1.2.1 Systemische juvenile chronische Arthritis (Still-Syndrom) 218

10.1.2.2 Seronegative $=$ kindliche Polyarthritis 222

10.1.2.3 Seropositive Polyarthritis = juvenile rheumatoide Arthritis 224

10.1.2.4 Oligoarthritis Typ I = frühkindliche Oligoarthritis 224

10.1.2.5 Oligoarthritis Typ II 225
10.1.2.6 Juvenile Spondarthritis

(Sonderform, in der Ilar-Klassifikation

nicht enthalten) 226

10.1.2.7 Juvenile Arthritis psoriatica (Sonderform, in der Ilar-Klassifikation nicht enthalten) 226

10.2 Seronegative Spondarthritiden und reaktive Arthritiden 229

H.M. BonÉL, G.M. LINGG

10.2.1 Spondylitis ankylosans 229

10.2.2 Osteoarthropathia psoriatica 244

10.2.3 Enteropathische Arthritiden 253

10.2.4 Akquiriertes Hyperostosesyndrom (SAPHO) 255

10.2.5 Reaktive Arthritiden nach intestinalem oder urogenitalem Infekt 258

10.2.6 Rheumatisches Fieber 260

10.3 Kollagenosen 261

H.M. BONÉL, G.M. LINGG

10.3.1 Systemischer Lupus erythematodes 261

10.3.2 Systemische Sklerose 265

10.3.3 Polymyositis und Dermatomyositis 270

10.3.4 Mischkollagenose 274

10.3.5 Vaskulitiden 277

Literatur Abschn. 10.1, 10.2 und 10.3281

$10.4 \quad$ Kristallarthropathien und assoziierte Erkrankungen 287

J. HodLER

10.4.1 Kalziumpyrophosphatdihydrat-

Kristallarthropathie 287

10.4.2 Hydroxyapatit-Kristallopathie 289

10.4.3 Gicht 291

10.4.4 Hämochromatose und Morbus Wilson 293

10.4.4.1 Hämochromatose 293

10.4.4.2 Morbus Wilson 294

10.4.5 Ochronose 295

Literatur 296

10.5 Andere Arthropathien 298

F. KaINBERger, K. Trieb

10.5.1 Neurogene Osteoarthropathie (inklusive diabetische Osteo-

arthropathie) 298

10.5.2 Rezidivierende Polychondritis 304

10.5.3 Akromegalie und hypophysärer Gigantismus 305

10.5.4 Hämophiliearthropathie 308

10.5.5 Multizentrische Retikulohistiozytose 312 Literatur 313

10.6 Arthrose (degenerative

Gelenkerkrankungen) 314

H. IMHOF

10.6.1 Definition 314

10.6.2 Epidemiologie 318

10.6.3 Pathophysiologie 319

10.6.3.1 Hyaliner Knorpel 319

10.6.3.2 Subchondralregion 321 
10.6.3.3 Meniskus/Diskus 321

10.6.3.4 Spezielle Pathophysiologie 323

10.6.4 Bildgebende Diagnostik 330

10.6.5 Hereditäre Arthrosen (hereditäre Chondroarthropathien) 333

10.6.5.1 Primär generalisierte Arthrose 333

10.6.5.2 Familiäre Kalziumpyrophosphaterkrankung 333

10.6.5.3 Familiäre Hydroxyapatiterkrankung 334

10.6.5.4 Chondrodysplasien 334

10.6.6 Spezielle Gelenke 335

10.6.6.1 Sternoklavikulargelenk 335

10.6.6.2 Schultergelenk 335

10.6.6.3 Ellbogengelenk 340

10.6.6.4 Handgrundgelenke und Interkarpalgelenke 341

10.6.6.5 Interphalangealgelenke und Metakarpophalangealgelenke 343

10.6.6.6 Hüftgelenk 344

10.6.6.7 Kniegelenk 348

10.6.6.8 Sprunggelenk 350

10.6.6.9 Intertarsalgelenke, Metatarsophalangealgelenke und Interphalangealgelenke 351 Literatur 352

10.7 Degenerative Wirbelsäulenerkrankungen 356

A. STÄBLER

10.7.1 Formen der Wirbelsäulendegeneration 356

10.7.2 Sozioökonomische Aspekte 357

10.7.3 Übergangsstörungen 358

10.7.4 Bandscheibendegeneration (Osteo-)Chondrose - Spondylose 360

10.7.5 Erosive intervertebrale Osteochondrose 368

10.7.6 Schmorl-Knoten (intraspongiöse Knorpelhernien) 378

10.7.7 Bandscheibenvorfall 387

10.7.8 Wirbelgelenkdegeneration 400

10.7.9 Spinalkanalstenose 405

10.7.10 Recessustenose 411

10.7.11 Neuroforamenstenose 412 Literatur 413

11 Bildgebende Diagnostik der Endoprothetik M. Krötz, S. Höpfner, K.-J. Pfeifer

11.1 Prinzip der Endoprothesen 419

11.2 Grundlagen der bildgebenden Diagnostik 420

11.2.1 Projektionsradiographie 420

11.2.2 Computertomographie 420

11.2.3 Nuklearmedizinische Diagnostik 420

11.2.4 Sonographie 420

11.2.5 Magnetresonanztomographie 420

\subsection{Spezieller Teil 421}

11.3.1 Schultergelenk 421

11.3.2 Hüftgelenk 422

11.3.3 Kniegelenk 423

11.3.4 Endoprothetik im Ellenbogenund Handbereich 424

11.3.5 Bildgebende Diagnostik der Komplikationen und Langzeitverlauf 424

Literatur 428

12 Erkrankungen der Weichgewebe U. SZEimies

12.1 Neuromuskuläre Erkrankungen 429

12.1.1 Myopathien 431

12.1.1.1 Progressive Muskeldystrophien 431

12.1.1.2 Metabolische Myopathien 433

12.1.1.3 Myotonien

(Ionenkanalerkrankungen) 435

12.1.1.4 Endokrine/toxische Myopathien 436

12.1.1.5 Myositiden 437

12.1.2 Störung der neuromuskulären Übertragung 437

12.1.3 Autoimmunerkrankungen mit neuromuskulärer Mitbeteiligung 437

12.1.4 Neuromuskuläre Syndrome 438

12.1.5 Neurogene Erkrankungen 438

12.1.5.1 Neuropathien, periphere Nervenläsionen 438

12.1.5.2 Radikulopathien 439

12.1.5.3 Plexuserkrankungen 439

12.1.5.4 Vorderhornzellerkrankungen 440

12.1.6 Muskelveränderungen nach Bestrahlung 442 Literatur 442

12.2 Weichteiltumoren 444

12.2.1 Allgemeiner Teil: Diagnostik, Staging, Strategien der Bildgebung 444 Literatur 450

12.2.2 Spezieller Teil 452

12.2.2.1 Weichteiltumoren des Fettgewebes 452

12.2.2.2 Weichteiltumoren des fibrösen Bindegewebes 454

12.2.2.3 Weichteiltumoren der Muskulatur 457

12.2.2.4 Weichteiltumoren der Lymphgefäße 459

12.2.2.5 Weichteiltumoren der Blutgefäße 461

12.2.2.6 Weichteiltumoren des peripheren Nervengewebes 464

12.2.2.7 Weichteiltumoren von unklarer zellulärer Abstammung 467

12.2.2.8 Weichteilmetastasen 468

12.2.2.9 Postoperative, posttherapeutische Bildgebung 469 Literatur 470 
12.3 Weichteilentzündungen 472

12.3.1 Autoimmunologisch induzierte Muskelund Weichteilentzündungen 472

12.3.1.1 Polymyositis 473

12.3.1.2 Dermatomyositis 473

12.3.1.3 Einschlusskörperchenmyositis 474

12.3.1.4 Fokale Myositis 475

12.3.1.5 Muskelsarkoidose 475

12.3.1.6 Eosiniphile Fasziitis 476
12.3.2 Erregerbedingte Muskelund Weichteilentzündungen 477

12.3.2.1 Erregerbedingte Myositiden 477

12.3.2.2 Weichteilentzündungen 479

12.3.3 Fremdkörperreaktionen 482

Literatur 484

Sachverzeichnis 487 


\section{Autorenverzeichnis}

Adam, Gerhard, Prof. Dr. med.

Klinik und Poliklinik für Diagnostische

und Interventionelle Radiologie

Radiologisches Zentrum

Universitätsklinikum Hamburg-Eppendorf

Martinistraße 52

20246 Hamburg

Baur-Melnyk, Andrea, Dr. med.

Institut für Klinische Radiologie

Abt. Röntgen B

Klinikum der Universität München, Großhadern

Marchioninistraße 15

81377 München

Bonél, Harald M., Dr. med.

Institut für Diagnostische Radiologie

Universität Bern

Freiburgstraße

3010 Bern

Schweiz

Erlemann, Rainer, Prof. Dr. med.

Chefarzt, AR AD NR

Institut für Radiologie

St. Johannes-Hospital

An der Abtei 7-11

47166 Duisburg

Freyschmidt, Jürgen, Prof. Dr. med.

Direktor der Radiologischen Klinik

Zentralkrankenhaus

St.-Jürgen-Straße

28205 Bremen

Hodler, JÜrg, Prof. Dr. med.

Chefarzt, Radiologie

Universitätsklinik Balgrist

Forchstraße 340

8008 Zürich

Schweiz
HÖPFNER, STEFAn, Dr. med.

Abteilung für Diagnostische Radiologie

Universitätsklinikum Gießen

Klinikstraße 36

35392 Gießen

Imhof, Herwig, Prof. Dr. med.

Universitätsklinik für Radiodiagnostik - Osteologie

Währinger Gürtel 18-20

1090 Wien

Österreich

Kainberger, Franz, Prof. Dr. med.

Universitätsklinik für Radiodiagnostik - Osteologie

Währinger Gürtel 18-20

1090 Wien

Österreich

KRötz, Michael, Dr. med.

Institut für Klinische Radiologie

Klinikum der Universität München, Innenstadt

Nußbaumstraße 20

80336 München

LINGG, GERWIN M., Dr. med.

Sana Rheumazentrum Rheinland-Pfalz AG

Zentrales Röntgeninstitut

Kaiser-Wilhelm-Straße 9-11

55543 Bad Kreuznach

Link, Thomas M., Prof. Dr. med.

Dept. of Radiology

University of California

400 Parnassus Ave (A-367)

Campus Box 0628

San Francisco, CA 94143

USA 
Nolte-Ernsting, C., Prof. Dr. med.

Klinik und Poliklinik für Diagnostische

und Interventionelle Radiologie

Radiologisches Zentrum

Universitätsklinikum Hamburg-Eppendorf

Martinistraße 52

20246 Hamburg

Pfeifer, K.-J., Prof. Dr. med.

Institut für Klinische Radiologie

Klinikum der Universität München, Innenstadt

Nußbaumstraße 20

80336 München

Rand, Thomas, Prof. Dr. med.

Radiodiagnostik und MR-Institut

Universitätskliniken

Lazarettgasse 14

1090 Wien

Österreich

Stäbler, Axel, Priv.-Doz. Dr. med.

Radiologie in München Harlaching

Grünwalder Straße 72

81547 München
Steinborn, Marc, Dr. med.

Institut für Diagnostische

und Interventionelle Radiologie

Abt. Kinderradiologie

Städtisches Krankenhaus München-Schwabing

Kölner Platz 1

80804 München

Szeimies, Ulrike, Dr. med.

Radiologie in München Harlaching

Grünwalder Straße 72

81547 München

Trieb, Klemens, Prof. Dr. med.

Abteilung Orthopädie

Klinikum Frankfurt/Oder

Müllroserchaussee 7

15236 Frankfurt/Oder 